\title{
Development of a Femur Neck Bone Mineral Density Measuring Device for Accurate Examination
}

\author{
Man-Seok Han ${ }^{1}$, Sun-youl Seo ${ }^{2 *}$, Yong-Kyun Kim ${ }^{3}$, Min-Cheol Jeon ${ }^{4}$, Hyun-kuk Lee, and Se-Jong Yoo $^{6}$ \\ ${ }^{1}$ Department Radiological Science, Kangwon National University, Samcheok 25913, Korea \\ ${ }^{2}$ Department of Radiology, Eulji University, Seongnam 13135, Korea \\ ${ }^{3}$ Department Radiotechnology, Daewon University, Jecheon 27135, Korea \\ ${ }^{4}$ Department of Diagnostic Radiology, Chungnam National University Hospital, Daejeon 35015, Korea \\ ${ }^{5}$ Department Nuclear Medicine, Eulji University Hospital, Daejeon 35233, Korea \\ ${ }^{6}$ Department Radiology, Konyang University Hospital, Daejeon 35365, Korea
}

(Received 10 May 2015, Received in final form 24 May 2016, Accepted 31 May 2016)

\begin{abstract}
In bone density examinations, a change in the measured BMD occurs owing to the differences between the measured areas. To address this problem, we aimed to develop a new auxiliary device that could be rotated by $15^{\circ}$ by fixing the ankle to the distal femur neck. Dual energy X-ray absorptiometry (DXA) of BMD examinations were performed once a year, but 10 patients were examined over three sessions to analyze the area for measuring the femur neck BMD. The goal of this test was to determine the device's reliability, and the results were expressed in terms of the standard deviation of measurements. After performing bone density measurements using the new auxiliary device on 10 normal patients, with three measurements for each patient, the obtained standard deviation was 0.03 . The standard deviation of the measured BMD was 0.19 when using the currently existing auxiliary device, while the standard deviation of the measured BMD was 0.03 when using the new auxiliary device. By using the new auxiliary device, the standard deviation could be reduced by $\sim 80 \%$. Accurate rotation of the femur neck was possible in all examinations, and the standard deviation of BMD measurements could be reduced by up to $80 \%$ compared with the measurements performed using the currently existing auxiliary device. We hope that this advantageous new design can be used as a standard auxiliary device for measuring the femur neck BMD.
\end{abstract}

Keywords : BMD, DXA, new auxiliary device, femur neck

\section{Introduction}

Osteoporosis is the most common bone metabolic disease, defined as a significantly lower bone mass in affected individuals compared with normal individuals of equivalent age and sex [1]. In addition, bone mineral density (BMD) is currently used in clinical diagnoses of osteoporosis as the most useful criterion for evaluating reactions associated with the rate of loss or gain of the bone material and for evaluating the results of therapeutic interventions [2]. Moreover, femur neck bone fractures are most common among elderly women, occurring owing to a low-energy damage and reduction in the BMD owing to aging, which are noted as the main causes of this type

(C)The Korean Magnetics Society. All rights reserved.

*Corresponding author: Tel: +82-31-740-7245

Fax:+82-31-740-7245, e-mail: angio7896@naver.com of fracture [3, 4]. Dual energy X-ray absorptiometry (DXA), which is most commonly used for measuring bone density, measures the BMD in hip joints, hips, and antecubital fossa, in which the body fat of the entire body can also be measured. This device has an outstanding resolution, is easy and efficient to operate, and is characterized by a low exposure rate [5]. A precision problem, which is recognized as a disadvantage, mainly occurs owing to the examinee's posture, the tool used for measuring bone density, and the examiner [6]. Proper posture of patients and accurate analysis of scans are the most significant factors determining accurate evaluation. High measurement accuracy prevents misdiagnoses and validates BMD research. When BMD measuring devices are used with the purpose of diagnosing osteoporosis or for evaluating the risk of fractures based on standard values, achieving high accuracy becomes crucial. Accuracy errors are expressed as root mean square standard deviation 
(RMS_SD) $\mathrm{g} / \mathrm{cm}^{2}$ or coefficient of variation (CV) \% and are characterized by different categories of RMS_SD. RMS_SD is a measure recommended by the international society for clinical densitometry (ISCD) for reporting the accuracy error. The calculated accuracy errors, (that is, the $\mathrm{CV}$ and \%) were previously reported for the pelvical (0.8-1.69\%), lumbar (1.0-1.2\%), femoral collum (1.11$2.2 \%)$, and trochanteric femoral (1.16-1.5\%) regions [7]. As a measure of overall accuracy, the RMS_SD was calculated as follows, where $\mathrm{m}$ denotes the number of patients.

$$
R M S \_S D=\sqrt{\sum_{j=1}^{m} S D_{j}^{2} / m}
$$

To express the measurement accuracy in terms of the CV measure, the RMS_CV measure, using CV instead of $\mathrm{SD}$ for individual patients, was calculated as

$$
\text { RMS_CV }=\sqrt{\sum_{j=1}^{m} C V_{j}^{2} / m}
$$

The present study was conducted with the overall goal of improving the reproducibility of examination posture of examinees, which affects accuracy. An auxiliary device that allows an internal rotation by up to $15^{\circ}$ was used in hip joint measurements. Currently existing auxiliary devices fix the patient's feet by an internal rotation of $15^{\circ}$, such that the extent of the femur neck rotation may vary depending on the patient's age and gender, causing examination-dependent variations in the area of the measured bone density. In bone density examinations, a change in the measured BMD occurs owing to the differences between the measured areas. To address this problem, we aimed to develop a new auxiliary device that could be rotated by $15^{\circ}$ by fixing the ankle to the distal femur neck.

\section{Materials and Methods}

\subsection{A method for measuring the femur neck BMD}

The hip and lumbar parts are routinely examined in BMD measurements. In the present study, only the hip was measured. dual energy X-ray absorptiometry (DXA, HOLOGIC, USA) was used as the bone density measuring device. As shown in Fig. 1, the hip bone density is measured while a patient lies with both feet internally rotated by $15^{\circ}$ using the existing auxiliary device. The inspection center is fixed for scanning. The region of interest (ROI) is defined using the obtained image. Bone mapping is performed after defining the ROI. From the obtained bone map, regions deviating from the bone are deleted and missing parts are filled in the map. When the bone map is set, the midline, neck box, Ward's triangle, and trochanter line are adjusted to obtain measurements.

Figure 2(A), (B) shows the bone density measurement results obtained using DXA.

\subsection{The area for measuring the BMD of the femur} neck using the existing auxiliary device

Regarding BMD examinations, 40 patients were examined once a year for a period of 5 years, to analyze the area for measuring the bone density of the femur neck. Standard deviations of bone density values are shown.

\subsection{Blueprint of the novel auxiliary device}

Figure 2(A) shows the plate that supports the feet to the distal femur neck fixation device. The area of the ankle region is $200 \mathrm{~mm}$, the distal femur is $100 \mathrm{~mm}$ with a length of $680 \mathrm{~mm}$, and $230 \mathrm{~mm}$ to the right side is bent upward at the angle of $75^{\circ}$ toward the foot sole. In addition, there is an oval-shaped hole on the top side that

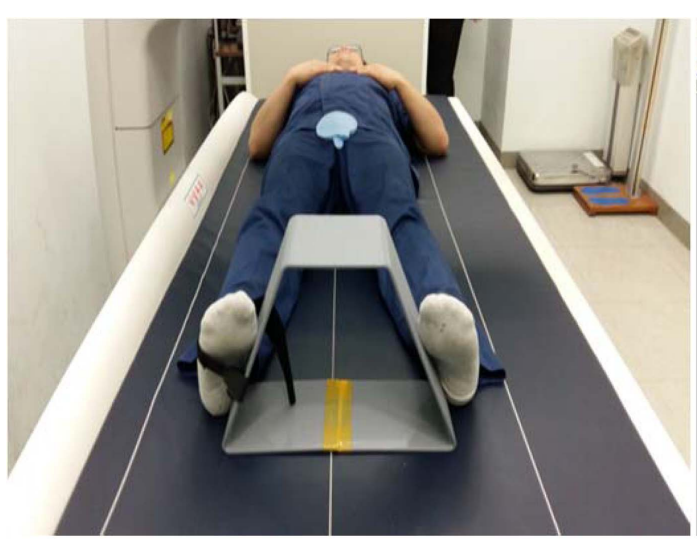

(A)

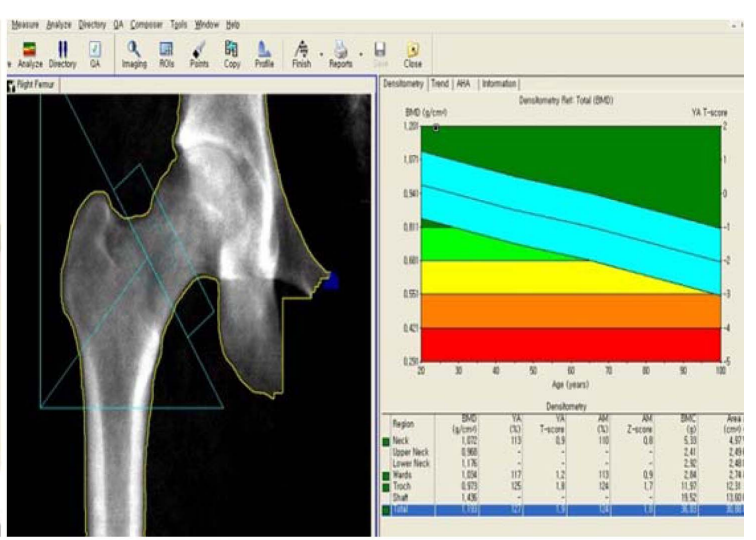

(B)

Fig. 1. (Color online) (A) Fixation of feet using the existing auxiliary device, (B) Image of the measured bone density of the femur neck and the resulting values. 


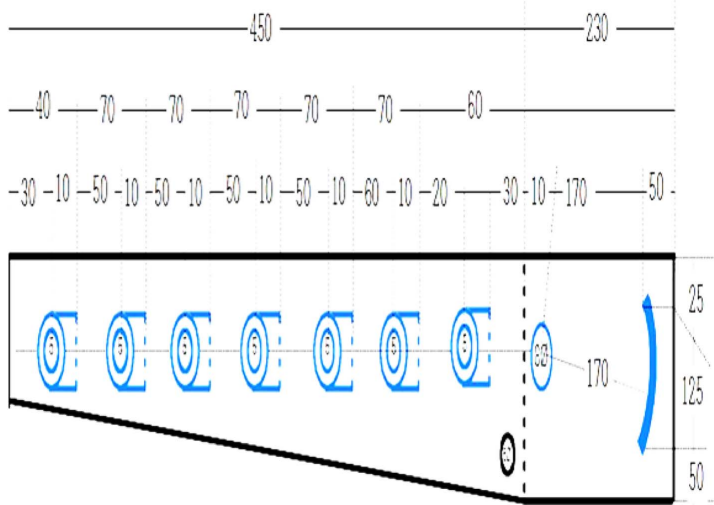

(A)

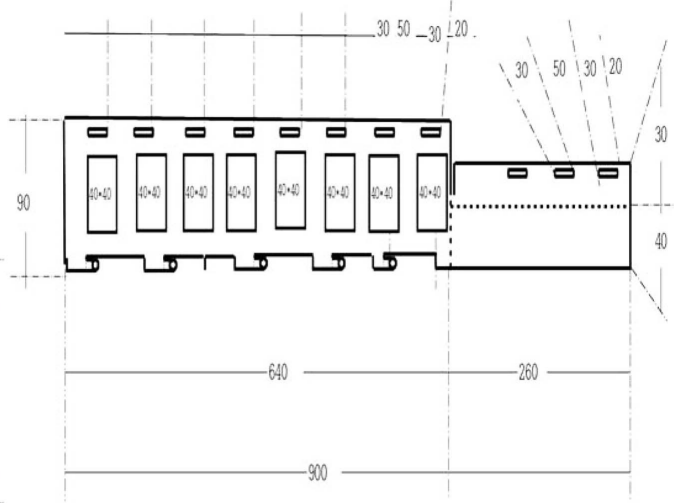

(B)

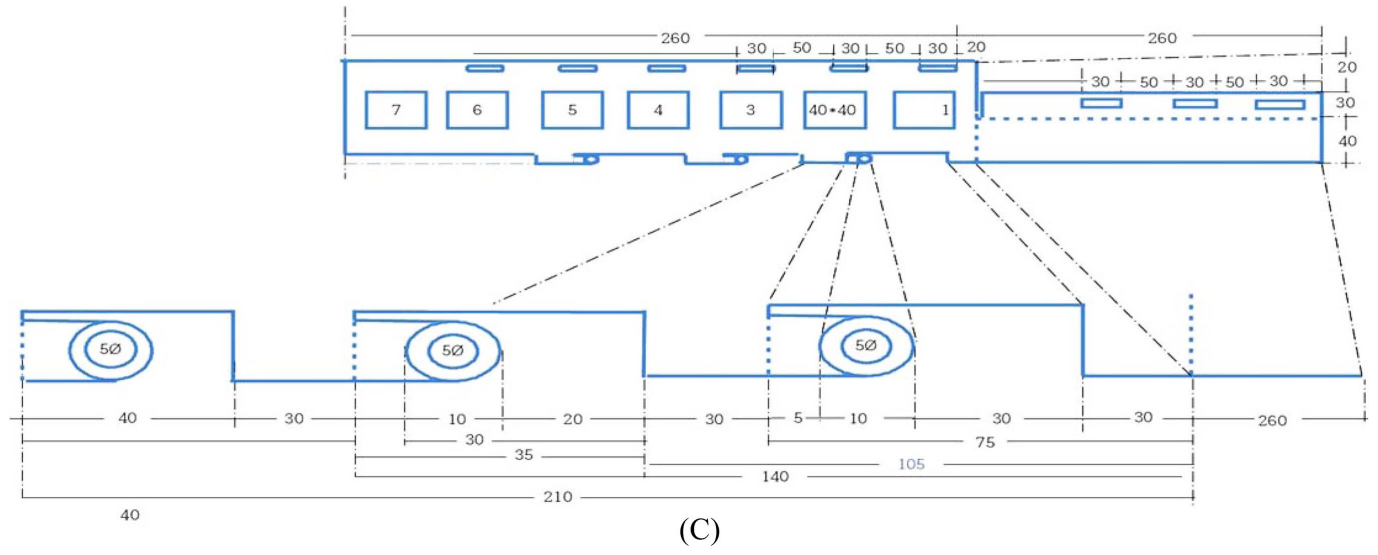

Fig. 2. (Color online) (A) Bottom face (left), (B) Fixation (left), (C) Detailed blueprint of the component in Figure (B).

can be used to measure and fix the angle while internally rotating the femur neck fixation device. There are seven 5-mm-diameter holes at the distance of $40 \mathrm{~mm}$ toward the interior of the $450-\mathrm{mm}$-long support plate that enables bending at $90^{\circ}$, and these holes are connected to the femur neck fixation device. The 5-mm-diameter holes inside the ankle region of the 450-mm-long support plate are required for assembling the left and right support plates and are used for adjusting the area of the femur neck region on the support plate based on the ankle; once the left and right plates are assembled (the left and right sides are identical).

Figure 2(C) schematically shows the direct fixation of the foot and distal femur neck region after assembly of the seven holes. The overall length of this component is $900 \mathrm{~mm}$, of which the 260 -mm-long segment on the right side is bent by $75^{\circ}$ upward, and there are three $0.5 \mathrm{~mm} \times$ $30 \mathrm{~mm}$ rectangular holes on the outer side, as shown in Figure 4. On the $640 \mathrm{~mm} \times 90 \mathrm{~mm}$ plate, there are five 5$\mathrm{mm}$-diameter circular holes and eight $0.5 \mathrm{~mm} \times 30 \mathrm{~mm}$ rectangular holes for fixing rubber bands on the outer side, and the plate is bent by $45^{\circ}$ relative to the $40-\mathrm{mm}$ - wide outer spot for fixing the ankle and distal femur neck. The eight $40 \mathrm{~mm} \times 40 \mathrm{~mm}$ square holes in the middle are designed to reduce the weight of the auxiliary device (the left and right sides are identical).

2.4. Standard deviation associated with measurements of the BMD of the femur neck using the new auxiliary device

BMD examinations were performed once a year, but 10 patients were examined over three sessions to analyze the area for measuring the femur neck BMD. The goal of this test was to determine the device's reliability, and the results were expressed in terms of the standard deviation of measurements.

\section{Results}

3.1. The area for measuring the BMD of the femur neck using the existing auxiliary device

Using the existing auxiliary device, the standard deviation of the BMD of 40 patients who were examined three or more times for 5 years was 0.19 . 


\subsection{Manufacturing of the new auxiliary device}

The internal rotation axis of the leg fixation plate in Fig. 3(A) can be rotated using a bolt. Because the range of the angle adjustment interval may vary, the maximum of the internal side was set to $40^{\circ}$ and the maximum of the external side was set to $10^{\circ}$, with $5^{\circ}$ intervals, to achieve equivalent rotation on both sides. The foot fixation plate brings the foot sole in flat contact with the device, and the left and right fixation bands on the leg fixation plates and leg fixing rubber bands are used to tightly fix both feet.

The bottom plate of the leg fixation plate in Fig. 3(B) supports the rotation of the leg fixation plates. Legs can be firmly fixed by using the rubber band hangers and leg fixation area adjustment axis. The femur neck angle adjustment bolt can be adjusted depending on the size of the angle between the areas of both femur necks, to accommodate the variation in the pelvis size across patients.

Figure 3(C) shows the lateral view of the completed auxiliary device fitted with 10 rubber bands and hooks that can fix the ankle to the distal femur neck. In this design, rotation that was previously restricted only to the ankle can now be performed in uniform fashion from the top of the foot to the knee, making the femur neck

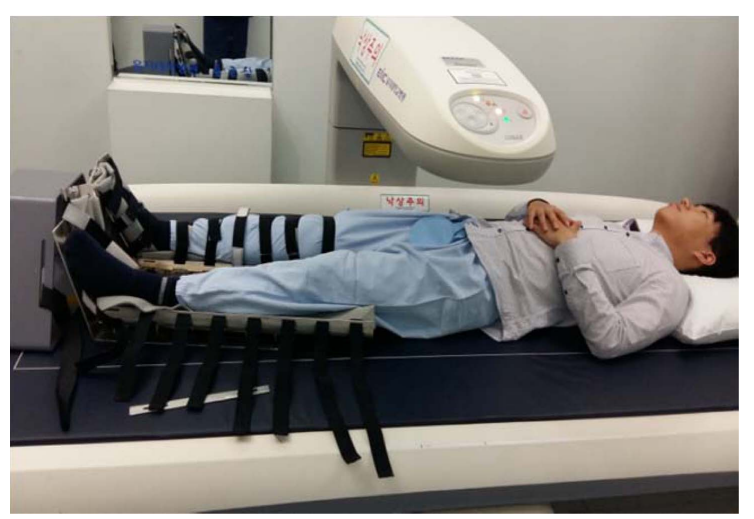

Fig. 4. (Color online) A new auxiliary device is used for measuring the femur neck BMD.

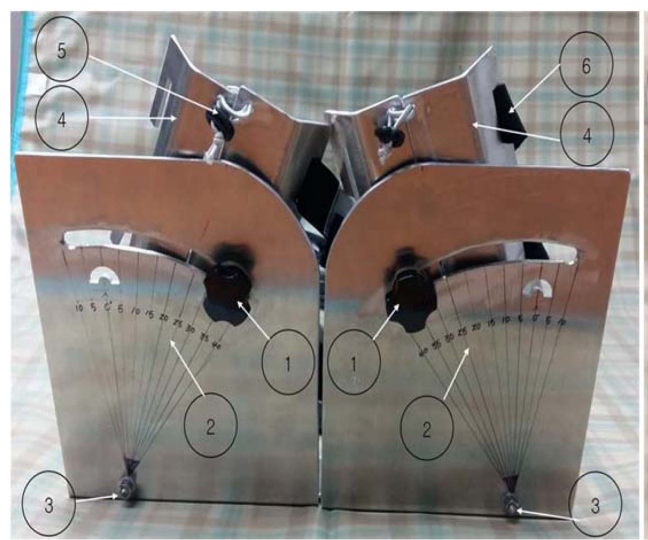

(A)

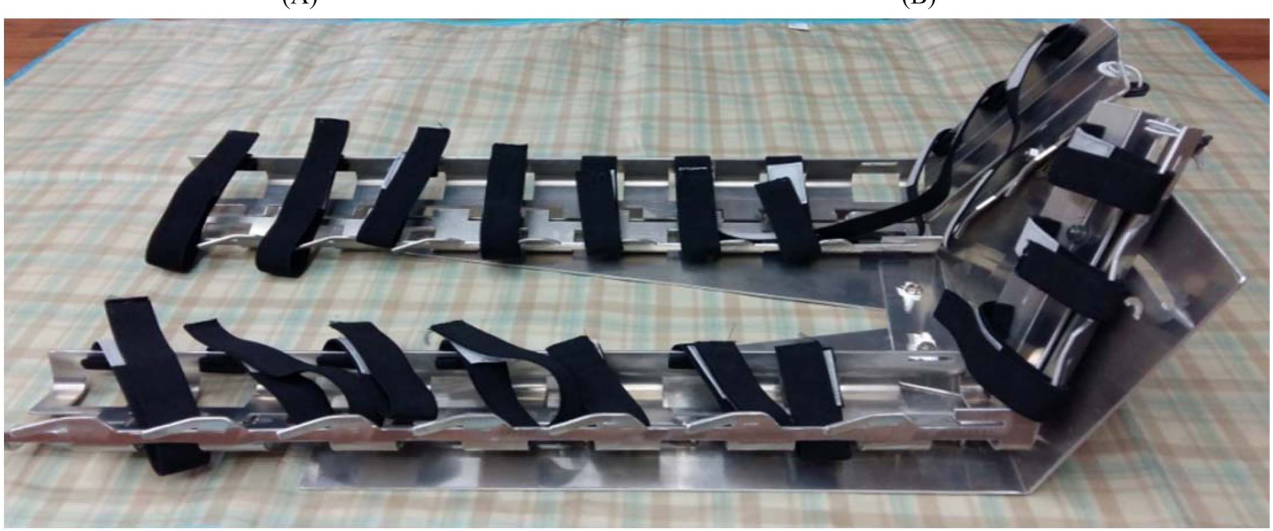

(C)

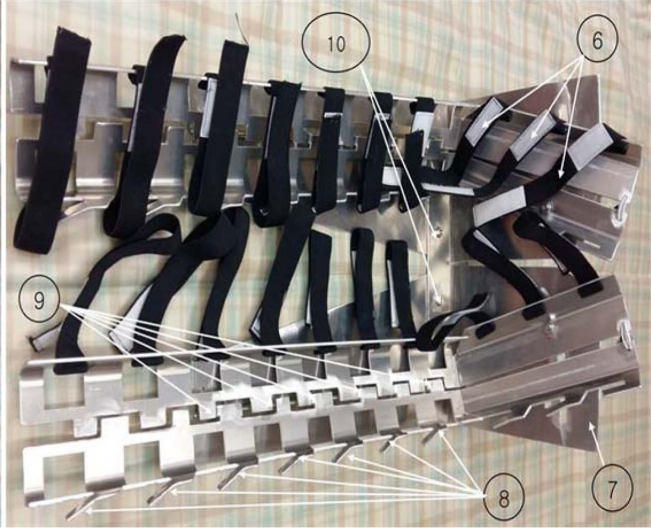

(B)

\section{.}


rotations consistent across different examinations.

The new auxiliary device was used for measuring the distal femur neck using the BMD measuring device (DXA), as shown in Fig. 4.

\subsection{Measuring the femur neck BMD using the new auxiliary device}

After performing bone density measurements using the new auxiliary device on 10 normal patients, with three measurements for each patient, the obtained standard deviation was 0.03 .

The standard deviation of the measured BMD was 0.19 when using the currently existing auxiliary device, while the standard deviation of the measured BMD was 0.03 when using the new auxiliary device. By using the new auxiliary device, the standard deviation could be reduced by $\sim 80 \%$.

\section{Discussion}

Proximal femur neck fracture due to severe injury may occur in all age groups, but fractures due to minor injury or without any clear association with injury mainly occur in elderly with osteoporosis [8-10] Osteoporosis is determined by the T-score that is used as a WHO diagnosis criterion in bone density examinations. Depending on the T-score, osteoporosis is categorized into three groups: $\mathrm{T} \geq$ -1.0 (normal), $-1.0 \leq \mathrm{T} \leq-2.5$ (osteopenia), and $\mathrm{T} \leq$ -2.5 (osteoporosis). The examination posture of patients and selection of ROIs of examinees significantly affect the outcomes of bone density examinations [11] Several studies have addressed the ROIs of examinees, but only a few studies addressed the posture of patients. In the present study, we addressed issues of reproducibility of the patients' posture in different examinations. Goh et al. recommended internal rotation by $\sim 15^{\circ}$ by considering anteversion when measuring bone density in the femur neck [12] Worldwide, bone density measurements of the femur neck are currently performed by internally rotating the femur neck by $15^{\circ}$. The existing auxiliary device that enables the $15^{\circ}$ rotation is a diamond-shaped device with a $28^{\circ}$ slope, in which only the ankle is internally rotated by $28^{\circ}$, which is insufficient for ensuring that the femur neck will be rotated by $15^{\circ}$. In addition, there is the disadvantage of low reproducibility of measurement posture across different examinations. Therefore, a novel auxiliary device was developed to rotate not only the ankle, but also the distal femur neck, for enabling uniform rotation. Reproducibility of examination postures can be improved by using the novel auxiliary device, and both femur necks can be rotated by precisely $15^{\circ}$. This is because the novel auxiliary device allowed to adjust the angle in steps of $5^{\circ}$, allowing examiners to accurately rotate the femur neck using this scale.

The major limitation of the present study was its relatively small sample size (only 10 healthy subjects). We believe that studies involving larger-size experimental groups must be conducted in the future.

\section{Conclusion}

When measuring bone density, readings vary depending on the femur neck rotation. Therefore, measurement postures must be consistent across different examinations. A novel auxiliary device was developed in this study to address this problem. The novel auxiliary device fixes both ankles and knees at the same time during rotation. As a result, accurate rotation of the femur neck was possible in all examinations, and the standard deviation of BMD measurements could be reduced by up to $80 \%$ compared with the measurements performed using the currently existing auxiliary device. We hope that this advantageous new design can be used as a standard auxiliary device for measuring the femur neck BMD.

\section{Acknowledgments}

This study was supported by 2013 Research Grant from Kangwon National University.

\section{References}

[1] W. J. Lee and N. W. Kim, Korea Society of Radiological Science 28, 227 (2005).

[2] D. Y. Kim, Korean J. Nucl. Med. 38, 275 (2004).

[3] B. L. Riggs and L. J. Melton, Am. J. Med. 75, 899 (1983).

[4] X. Cheng, J. Li, Y. Lu, J. Keyak, and T. Lang, Bone J. 40, 169 (2014).

[5] J. S. Choi, G. C. Ahn, and C. S. Lee, Jouranl of Korean Society of Spine Surgery 10, 75 (2003).

[6] Y. H. Kang and K. H. Jo, Korea Society of Radiological Science 29, 229 (2006).

[7] International Society for Clinical Densitometry (ISCD).

[8] A. Naimark, J. Kossoff, and A. Schepsis, AJR Am. J. Roentgenol. 133, 889 (1979).

[9] H. F. Newton-John and D. B. Morgan, Clin. Orthop. Relat. Res. 71, 229 (1970).

[10] B. E. Nordin, Calcif. Tissue. Int. 40, 57 (1987).

[11] Y. H. Lee, I. J. Lee, and H. J. Yang, Korea Society of Radiological Science 35, 1 (2012).

[12] J. C. H. Goh and S. L. Low, Calcif. Tissue. Int. 57, 340 (1995). 